\title{
Relationships among Child Maltreatment, Picture Completion Test, and Posttraumatic Symptoms: Two Examinations Using WISC-III for Japanese Children
}

\author{
Kohske Ogata ${ }^{1,2}$ \\ ${ }^{1}$ Graduate School for Creative Cities, Osaka City University, Osaka, Japan \\ ${ }^{2}$ Osaka Prefectural Government Higashiosaka Child-Family Center, Osaka, Japan \\ Email:koh-ske@sakai.zaq.ne.jp \\ Received May $18^{\text {th }}, 2012$; revised June $20^{\text {th }}, 2012$; accepted July $13^{\text {th }}, 2012$
}

\begin{abstract}
The aim of the present studies was to confirm a relationship among child maltreatment, Picture Completion (PC) test, and posttraumatic symptoms in Japanese maltreated children. In some previous researches, it was found that maltreated children scored higher on the PC test within the Wechsler series subtests. It is hypothesized that the relative higher score of maltreated children may be an indicator of hyperarousal symptoms in posttraumatic stress disorder (PTSD). In prior studies, there were some weaknesses on methodology. They had problems with a small sample size and indirect methods to test the hypothesis. In study I, the prior finding was replicated through the same indirect methodology as previous studies, using a larger sample size. The participants were 490 Japanese children (170 maltreated and 320 controls) sampled from the records of Child Guidance Centers in Japan. Results showed that a significant difference was found only for the PC score in all the subtests in the Wechsler Intelligence Scale for Children third edition $(F[1,487]=12.6, p<.001)$, replicating the previous finding using a larger sample. In study II, the hypothesis was verified directly using another sample set (40 maltreated). Only the correlation coefficient was significant between the deviated score on the PC test and the subscale of PTSD in Alternate version of the Trauma Symptom Checklist for Children ( $r=.33$ for all subtests, $p<.05 ; r=.38$ for performance subtests, $p<.05$ ). It may be a robust finding that the relative higher performance on PC in maltreated children relates to hyperarousal symptoms in PTSD. The author concludes that the hypothesis in the prior researches was demonstrated through the present two studies.
\end{abstract}

Keywords: WISC-III; Child Maltreatment; Intelligence; Traumatic Stress

\section{Introduction}

Child abuse and neglect are enormous traumatic events for children and have various traumatic impacts on their development. There have been abundant researches, particularly examining their intellectual development. It is well known that the psychometric intelligence, defined as intelligence measured using an intelligence test, of maltreated children is lower than that of non-maltreated children (Ex. Buckle, Lancaster, Powell, \& Higgins, 2005; Delaney-Black, Covington, Ondersma, Nordstrom-Klee, Templin, Ager, Janisse, \& Sokol, 2002; Prasad, Kramer, \& Ewing-Cobbs, 2005). Findings that show a lower intelligence level have been established, whereas there is little information on subtest scatter and/or the intellectual profile of maltreated children.

Frankel, Boetsch, and Harmon (2000) studied the elevated Picture Completion (PC) score of 14 maltreated children. The researchers demonstrated that maltreated preschoolers scored significantly higher on the PC subtest of Wechsler Preschool and Primary Scale of Intelligence Revised (WPPSI-R) than the children's mean score of performance subtests and of all subtests. Frankel et al. suggested from their results that a higher score on the PC related to a hyper-vigilance symptom of posttraumatic stress disorder (PTSD) in maltreated children; however, their study had defects in the research design regarding both the small sample size and no comparison group. Ogata
(2011) replicated the finding using a Japanese sample. In the study, a comparison group $(n=12)$ was set and differences in the types of child maltreatment were analyzed; however, this investigation also had the weakness of a small sample size (12 sexually abused, 12 physically abused, 12 neglected, and 12 comparison). Nolin and Ethier (2007) investigated neglected and/or physically abused children using neuropsychological assessments. The unexpected results surprised the researchers by showing that neglected children $(n=28)$ scored significantly higher on problem-solving ability and abstract reasoning than non-maltreated $(n=53)$ and neglected with physical abuse $(n=$ 56) groups. These findings suggest that maltreated children may have a specific intellectual profile, probably influenced by their abuse and/or neglect experiences. Thus, to clarify the intellectual profile in maltreated children is considered to partially contribute to understanding their traumatic status. However, some pioneering researches have the problem on methodology.

The following two studies addressed the problems on methodology in the prior researches. In particularly, the hypothesis that the PC score may relate to the hyperarousal symptom in PTSD was examined.

\section{Study I}

The primary weakness in the prior researches involves the 
small sample size. A larger sample size is indispensable for generalization of the previous findings. The purpose of study I was to confirm the robustness of the findings relating to the intellectual profile of maltreated children. In study I, the prior finding that the PC scores of the maltreated children were higher than those of non-maltreated children was replicated using a larger sample size.

\section{Method}

The participants included 490 Japanese children (207 girls and 283 boys). The samples were retrospectively selected from the records of child guidance centers (CGC) in Osaka prefecture, in a region where the child maltreatment were reported most frequently, in Japan. The CGC, like Child Protective Services, are required to investigate child maltreatment reports, to protect children (under 18 years old) from further maltreatment, and to provide rehabilitative services to children, parents, and other family members involved. The children that participated were classified into two groups (170 maltreated and 320 comparisons) according to the CGC records, in which maltreatment was determined through review of the records. Mean age (in months) at maltreatment was $135.5(S D=32.0)$ and that of comparisons was $146.9(S D=30.5)$. The maltreated group consisted of 76 physically abused, 15 sexually abused, 26 psychologically abused, and 53 neglected children. The comparison group had been neither abused nor neglected according to the CGC records. Physical abuse consisted of bruises, burns, cuts, scratches, or bone fractures. Sexual abuse was defined in this study as attempted or actual sexual contact or interaction of any form between the participant and a caregiver or other responsible adult for purposes of the adult's sexual gratification. Sexual contact experiences ranged from fondling, genital touching, and masturbation of or by another person to attempted or completed vaginal intercourse. In this study, all psychologically abused children were eyewitnesses to domestic violence. Following Kotch, Lewis, Hussey, English, Thompson, Litrownik et al. (2008), neglect was composed of two subtypes: the first subtype, failure to provide, involves the failure of the caregiver or responsible adult to meet the minimum physical needs of the child; the second subtype, lack of supervision, occurs when the caregiver or responsible adult does not take sufficient, developmentally appropriate action to ensure the child's safety inside and outside the home setting. The comparison group consisted of children needing foster care, who needed to be admitted to child welfare institutions, with school maladaptation, and with personality problems alleged by their parents; however, they had not experienced any child maltreatment according to the information on the records obtained from their parents and themselves. All the CGC reports, whether substantiated or not, were considered reliable indicators of maltreatment in Japan.

The intellectual functioning of children was assessed by 10 core subtests of the Wechsler Intelligence Scale for Children third edition (WISC-III). A child's cognitive development was measured by the WISC-III Japanese version; the author confirmed from the case files that a trained child psychologist had administered the WISC-III to the child in a CGC. The intelligence test was administered after informed consent by their parents or caregivers, and/or themselves. The scale has been standardized on a national sample of 1125 children ranging in age from 5 through 16 years and 11 months. Psychometric traits of the WISC-III Japanese version were adequate on reliability and validity (Wechsler, 1991/1998). Reliability coefficients, based on split-half correlations, range from .64 to .85 for the 10 core subtests, and .95 for the full-scale intelligence quotient (FIQ); reliability coefficients, based on test-retest correlations, range from .54 to .89 for the 10 core subtests, and .93 for the FIQ. Construct validity was also confirmed using factor analysis; the four factor model was adopted, which is the same as the original Wechsler model.

The data were analyzed as follows. First, t-tests were used repeatedly to confirm that there were no differences between the maltreated and comparison groups on the 10 subtests. Next, multivariate analysis of covariance (MANCOVA) was used to examine the difference between the maltreated and comparison groups as controlled for the FIQ. Overall significance on MANCOVA was followed by Bonferroni-corrected ANCOVA. Statistical significance was set at $p<.05$, except for Bonferroni-corrected ANCOVA $(p<.005)$. Statistical analyses were performed using IBM SPSS 20 Japanese version.

\section{Results and Discussion}

All results of subtest profiles are summarized in Table 1. There was no difference in the FIQ between the groups (Maltreated $M=82.3, S D=14.3$; Comparison $M=83.5, S D=13.8$; $t[488]=.94, p=.35)$. To examine subtest scatters, it was necessary to compare the subtest profiles to control for the overall level of the FIQ. Thus, the FIQ was used as a covariate. MANCOVA revealed a significant difference in the WISC-III subtest results between the two groups (Wilks $\lambda=.92, F[10$, $478]=3.91, p<.001)$. The obtained results demonstrated that the intraindividual variability in the subtest profile of the maltreated children differed from that of the comparison group; therefore, further examinations are needed to identify the origin of the significant difference (Table 2).

A significant difference by Bonferroni-corrected ANCOVA $(p<.005)$ was found only for the PC subtest score $(F[1,487]=$ $12.6, p<.001)$. The adjusted score, controlled for the FIQ, of the maltreated $(M=7.9)$ was significantly higher than that of the comparison $(M=7.4)$ children. Differences in the other subtests failed to reach statistical significance.

The results were consistent with the findings of both Frankel et al. (2000) and Ogata (2011), showing a robustness of the

Table 1.

Subtest profiles and differences between maltreated and comparison children in study I.

\begin{tabular}{lcccccc}
\hline & \multicolumn{2}{c}{$\begin{array}{c}\text { Maltreated } \\
(n=170)\end{array}$} & \multicolumn{2}{c}{$\begin{array}{c}\text { Comparison } \\
(n=320)\end{array}$} & \multicolumn{2}{c}{$\begin{array}{c}\text { Difference } \\
\text { between }\end{array}$} \\
\hline Subtests & $M$ & $S D$ & $M$ & $S D$ & $t$ & $p$ \\
\hline Picture completion & 7.8 & 2.4 & 7.4 & 2.4 & 1.91 & .057 \\
Information & 7.0 & 2.8 & 7.5 & 2.8 & 1.77 & .077 \\
Coding & 7.9 & 2.9 & 8.2 & 2.8 & .89 & .374 \\
Similarities & 7.2 & 3.0 & 7.6 & 2.9 & 1.42 & .155 \\
Picture arrangement & 7.1 & 3.1 & 7.6 & 2.7 & $1.85^{\mathrm{a}}$ & .066 \\
Arithmetic & 7.2 & 2.8 & 7.7 & 2.7 & 1.87 & .062 \\
Block design & 8.0 & 3.0 & 8.3 & 3.4 & 1.23 & .218 \\
Vocabulary & 7.4 & 2.6 & 7.4 & 2.9 & .09 & .924 \\
Object assembly & 7.4 & 3.2 & 7.9 & 2.7 & $1.82^{\mathrm{a}}$ & .070 \\
Comprehension & 8.0 & 2.7 & 8.0 & 2.9 & .11 & .916
\end{tabular}

Note: Student's $t$-test, $\mathrm{d} f=488,{ }^{\mathrm{a}}$ Welch's $t$-test. 
Table 2.

ANCOVAs controlled for the FIQ as a covariate in study I.

\begin{tabular}{lcccccccc}
\hline \multirow{2}{*}{ Subtests } & \multicolumn{2}{c}{ Equivalent slope across the groups } & \multicolumn{2}{c}{ Regression significance } & \multicolumn{2}{c}{ Levene test } & \multicolumn{3}{c}{ ANCOVA } \\
\cline { 2 - 9 } & $F$ & $p$ & $F$ & $p$ & $F$ & $p$ & $F$ & $p$ \\
\hline Picture Completion & 1.30 & .255 & 455.44 & $.000^{*}$ & 1.34 & .248 & 12.63 & $.000^{*}$ \\
Information & .58 & .446 & 448.56 & $.000^{*}$ & .40 & .525 & 2.39 & .123 \\
Coding & .00 & .977 & 204.37 & $.000^{*}$ & .01 & .911 & .20 & .654 \\
Similarity & 2.07 & .151 & 467.16 & $.000^{*}$ & 2.24 & .135 & 1.15 & .285 \\
Picture Arrangement & 6.32 & .012 & 344.36 & $.000^{*}$ & .55 & .458 & 2.92 & .088 \\
Arithmetic & 4.34 & .038 & 528.49 & $.000^{*}$ & 13.15 & $.000^{*}$ & 2.96 & .086 \\
Block Design & 3.24 & .073 & 401.39 & $.000^{*}$ & 2.27 & .132 & .66 & .419 \\
Vocabulary & 6.84 & .009 & 466.25 & $.000^{*}$ & 1.01 & .316 & .62 & .432 \\
Object Assembly & 3.45 & .064 & 315.01 & $.000^{*}$ & 2.02 & .156 & 2.90 & .089 \\
Comprehension & 1.46 & .228 & 277.18 & $.000^{*}$ & .41 & .522 & .33 & .564 \\
\hline
\end{tabular}

Note: Statistical significance level set at $.005(.05 / 10)$, representing with the symbol*.

finding that the PC test score of maltreated may be higher than that of comparison children. An adequate sample size $(n=490)$ may strongly support the evidence. The larger sample size in the present study, approximately tenfold that in previous studies, would add robustness to the previous finding. In addition to the adequate sample size, using a comparison group could enhance the validity of the findings.

\section{Study II}

Study I replicated the previous researches. The relative higher PC score in maltreated children was obtained using a larger sample size; however, the methodology may have another problem for examining the hypothesis suggested in the discussion of Frankel et al. (2000). The hypothesis involved the relationship between a deviated PC score, from the mean score of all/performance subtests, and hyperarousal symptoms of PTSD in maltreated children. To elucidate the hypothesis, bivariate correlation needs to be confirmed between the PC score and the measure on the PTSD. Therefore, study II did not examine indirect evidence that maltreated children scored higher on the PC subtest than the average subtest score, but direct evidence of the correlation coefficient between the two variables.

\section{Method}

Participants included 40 maltreated children aged (in months) $73-197(M=143, S D=30), 26$ boys and 14 girls who had experienced physical abuse $(n=16)$, sexual abuse $(n=2)$, psychological abuse $(n=6)$, or neglect $(n=16)$. Data were elicited from the same CGC as in study I. They were assessed to evaluate cognitive functioning using the WISC-III and to measure posttraumatic symptoms using an Alternate version of the Trauma Symptom Checklist for Children (TSCC-A). Following or preceding the WISC-III, the TSCC-A was administered after informed consent by their parents or caregivers, and/or themselves.

The Japanese version of TSCC-A was standardized for 1698 children. Psychometric traits were as follows. On internal consistency, Cronbach's alpha was .81 for anxiety, .78 for depression, .86 for anger, .84 for PTSD, and .78 for dissociation. The validity was examined using 787 children in child welfare in- stitutions. Correlations between 33 items on the trauma-related child behavior checklist and TSCC-A were as follows:.14 for anxiety, .20 for depression, .25 for anger, .15 for PTSD, and .15 for dissociation (Briere, 1996/2009).

Data were analyzed as follows. After deviation score of PC was calculated, correlation coefficients between deviated PC score and TSCC-A were computed. Statistical analyses were performed using IBM SPSS 20 Japanese version.

\section{Results and Discussion}

Results of the two assessments of the participants are shown in Table 3. Their FIQ was $89.5(S D=12.5)$ and performance IQ was $90.4(S D=12.6)$. Following Frankel et al. $(2000)$, the deviation of PC scores from all or performance subtests were calculated. Correlation coefficients between the deviated PC score and the subscales of TSCC-A were shown in Figure 1. Of five subscales, only the PTSD measure was correlated with PC scores deviating from the average of both all and performance subtests.

Table 3.

Descriptive statistics on the WISC-III and TSCC-A in study II.

\begin{tabular}{lcc}
\hline & $M$ & $S D$ \\
\hline WISC-III & 8.3 & 2.2 \\
Picture Completion & 7.9 & 2.3 \\
Information & 9.1 & 3.2 \\
Coding & 8.4 & 2.8 \\
Similarities & 8.6 & 3.3 \\
Picture Arrangement & 8.5 & 2.9 \\
Arithmetic & 8.7 & 3.2 \\
Block Design & 8.7 & 2.3 \\
Vocabulary & 8.4 & 2.7 \\
Object Assembly & 9.0 & 2.7 \\
Comprehension & & \\
TSCC-A & 57.1 & 9.0 \\
Anxiety & 54.9 & 10.7 \\
Depression & 52.4 & 9.2 \\
Anger & 57.2 & 8.7 \\
PTSD & 54.4 & 9.9 \\
Dissociation & & \\
\hline
\end{tabular}




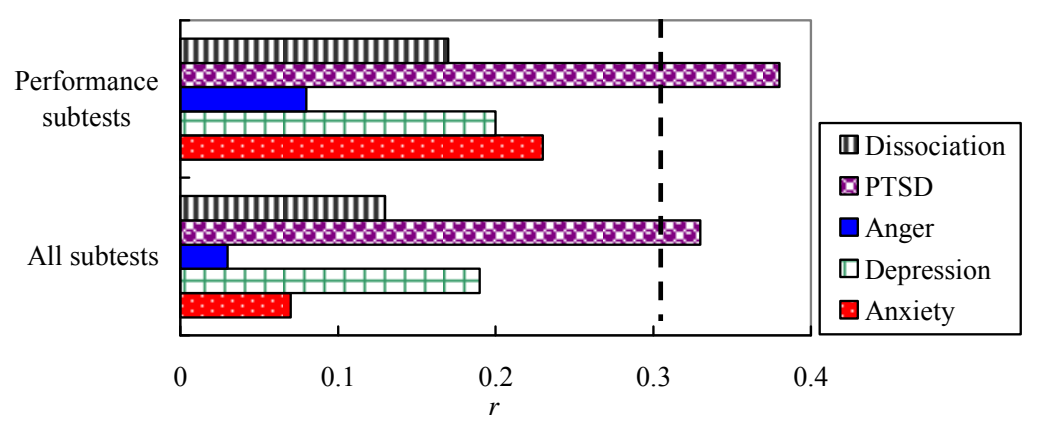

Figure 1.

Correlation coefficients between deviated PC score from all/performance subtests and subscales of TSCC-A. Dotted line represents statistical significance at $p<.05$.

The results may directly support the hypothesis suggested in Frankel et al. (2000) that only the PTSD scale correlated with the deviated PC score. The present findings could be evidence directly examining the relationship between the PC subtest and posttraumatic symptoms. In addition, results showing that the other subscales did not correlate with the deviated PC score suggest a discriminative validity for the higher PC score being an indicator of the hyperarousal symptom.

However, study II may also have a limitation. Significant correlation coefficients were found, but the PTSD subscale of TSCC-A involved all aspects of posttraumatic symptoms: intrusion, avoidance, and hyperarousal. Thus, it is possible that this finding suggests a relation among the other PTSD symptoms. Future research needs to measure the PTSD symptoms separately and to confirm that the higher PC score relates to the hyperarousal symptom rather than other symptoms.

\section{Total Discussion}

The present studies provide and add to evidence for the finding that maltreated children scored higher on the PC subtest because of a relationship between the PC score and the hyperarousal symptom. The hypothesis suggested in Frankel et al. (2000) was verified through the two studies. In study I, a larger sample size was used to confirm the robustness of the prior finding and, in study II, correlations were examined directly to verify the relationship between the deviated PC score and the measure of PTSD. The author concludes that the present report contributes to the body of literature on child abuse and neglect, particularly to understanding the diverse impact of child maltreatment on child development.

However, there are some differences from the prior findings. Ogata (2011) reported different effects of the types of maltreatment on the intellectual profile. Only sexually abused children scored higher on the PC test in the research. The design of Ogata's study adopted matching methodology. Group differences were readily detected in the matching design because their correlation coefficients were considered; however, the sample size of a matched design is likely to be small. The total participants in Ogata's study was 48 , whereas study I in the current report included 490 participants; therefore, this study did not focus on the type of maltreatment but paid attention to the overall robustness of the finding.

These findings might demonstrate the adverse and deleterious circumstance where maltreated children live. Because daily atmosphere in their family should influence on their emotional status strongly, they may suffer from hypervigilance and/or hyperarousal symptom.

There are some clinical implications. Firstly, in these analyses, controlling the FIQ was important because raw subtest scores between groups were not significantly different (Table 1). The results indicated that the intellectual characteristics of maltreated children were represented not as a raw score but as an adjusted score in the PC subtest, controlled for the overall level of the FIQ. Therefore, it may be useful in clinical settings to calculate the ratio (subtest score/FIQ), and it was recommended to evaluate intraindividual differences to assess children who have experienced maltreatment. Secondly, it was recommended to include the $\mathrm{PC}$ subtest in the test battery to assess cognitive functioning. The WISC-IV excludes the PC subtest from 10 core subtests in the current revision. As a supplemental subtest, the PC score is unnecessary to compute the FIQ; however, the author recommends the PC subtest for assessing maltreated children on the basis of the current findings. The PC test might be able to screen their hyperarousal symptoms in PTSD. A maltreated child who scores relative higher on the PC should be referred to a child psychiatrist for detailed diagnosis of PTSD. Whether or not maltreated children have symptoms related to the PTSD, their psychological treatment may vary according to their mental status. The author provides some recommendations from the view of the clinical setting.

Finally, the findings are discussed from cultural standpoints. The current investigations were conducted in Japan. Almost all previous researches were Western findings. Thus, the present findings replicating Western studies should be compared with other non-Western reports. For example, in the Turkish literature, Bulut and colleagues studied PTSD in children who have experienced natural disasters (Bulut, 2004, 2006, 2010; Bulut, Bulut, \& Tayli, 2005). Bulut (2010) reported from longitudinal investigations that overall PTSD rates in children gradually decreased. The research revealed gender differences that were detected in intrusion and avoidance symptoms. It is difficult to directly compare these findings with the current research because of the difference in the participants in each study.

\section{REFERENCES}

Briere, J. (2009). Trauma Symptom Checklist for Children (TSCC) Professional Manual. In S. Nishizawa, \& C. Yamamoto (Trans. and write). Odessa, FL: Psychological Assessment Resources.

Buckle, S. K., Lancaster, S., Powell, M. B., \& Higgins, D. J. (2005). The relationship between child sexual abuse and academic achievement in a sample of adolescent psychiatric inpatients. Child Abuse \& Neglect, 29, 1031-1047. doi:10.1016/j.chiabu.2004.12.013 
Bulut, S. (2004). Factor structure of posttraumatic stress disorder in children experienced natural disaster. Psychologia: An International Journal of Psychology in the Orient, 47, 158-168.

doi:10.2117/psysoc.2004.158

Bulut, S. (2006). Comparing the earthquake exposed and non-exposed Turkish children's Post Traumatic Stress Reactions. Anales de Psicología, 22, 29-36.

Bulut, S. (2010). Children's posttraumatic stress reactions and subsymptoms: Three years of a longitudinal investigation study after a direct exposure to the earthquake and school's collapse. Turk Psikoloji Dergisi, 25, 87-98.

Bulut, S., Bulut, S., \& Tayli, A. (2005). The dose of exposure and prevalence rates of post traumatic stress disorder in a sample of Turkish children eleven months after the 1999 Marmara earthquakes. School Psychology International, 26, 55-70. doi:10.1177/0143034305050893

Delaney-Black, V., Covington, C., Ondersma, S. J., Nordstrom-Klee, B., Templin, T., Ager, J., Janisse, J., \& Sokol, R. J. (2002). Violence exposure, trauma, and IQ and/or reading deficits among urban children. Archives of Pediatrics \& Adolescent Medicine, 156, 280-285.

Frankel, K. A., Boetsch, E. A., \& Harmon, R. J. (2000). Elevated pic- ture completion scores: A possible indicator of hypervigilance in maltreated preschoolers. Child Abuse \& Neglect, 24, 63-70. doi:10.1016/S0145-2134(99)00110-6

Kotch, J. B., Lewis, T., Hussey, J. M., English, D., Thompson, R., Litrownik, A. J., Runyan, D. K., Bangdiwala, S. I., Margolis, B., \& Dubowitz, H. (2008). Importance of early neglect for childhood aggression. Pediatrics, 121, 725-731. doi:10.1542/peds.2006-3622

Nolin, P., \& Ethier, L. (2007). Using neuropsychological profiles to classify neglected children with or without physical abuse. Child Abuse \& Neglect, 31, 631-643. doi:10.1016/i.chiabu.2006.12.009

Ogata, K. (2011). Intellectual profile of sexually abused children in Japan: An analysis of WISC-III subtests compared with physically abused, neglected, and non-maltreated children. Psychology, 2, 169172. doi:10.4236/psych.2011.23027

Prasad, M. R., Kramer, L. A., \& Ewing-Cobbs, L. (2005). Cognitive and neuroimaging findings in physically abused preschoolers. Archives of Diseases in Childhood, 90, 82-85. doi:10.1136/adc.2003.045583

Wechsler, D. (1998). Manual for the Wechsler intelligence scale for children-third edition. San Antonio: The Psychological Corporation. 